\title{
Géolocalisation en Intérieur par Ultrasons
}

\author{
G. BEAUGENDRE ${ }^{1}$, F. VINCENT ${ }^{2}$, M. BERRANGER ${ }^{3}$ \\ Département d'Electronique Optronique et Signal, ISAE, 1, Place Emile BLOUIN, Toulouse. \\ ${ }^{1}$ guillaume.beaugendre@isae.fr, ${ }^{2}$ francois.vincent@isae.fr, ${ }^{3}$ matthieu.berranger@isae.fr
}

RESUME : L'équipe d'électronique et de traitement du signal de l'Institut Supérieur de l'Aéronautique et de l'Espace (ISAE) a développé une maquette pédagogique permettant de se familiariser avec les systèmes de Géolocalisation. Celui-ci fonctionne en intérieur et reprend le principe de fonctionnement du système GPS réel. Les ondes émises sont ici ultrasonores et non électromagnétiques, ces signaux ont été simplifiés et les émetteurs sont fixes. Chaque groupe d'étudiants dispose d'un récepteur à ultrasons, de son électronique associée, d'une carte d'acquisition et d'un ordinateur équipé de Matlab $^{\circledR}$. L'objectif de ce projet est de calculer la position du récepteur dans une salle à partir des signaux reçus. Ce projet s'adresse à des étudiants d'un niveau Licence ou première année de Master ayant les connaissances de base en traitement du signal. Ils travaillent par groupes de 2 de manière semi-encadrée pendant une vingtaine d'heures.

Mots clés : GPS, géolocalisation, projet pédagogique, navigation, électronique, ultrasons, traitement du signal.

\section{INTRODUCTION}

L'ISAE, issue du rapprochement entre l'ENSICA et Supaéro, forme des ingénieurs spécialisés en aéronautique. Le projet présenté ici, est effectué en première année de formation. L'objectif est de consolider les bases scientifiques des élèves lors du premier semestre pour mieux aborder les sciences de l'ingénieur et les disciplines plus spécifiques du domaine aéronautique et spatial. Au sein de l'Unité de Formation Avioniques et Système (UFAS), les étudiants sont amenés à étudier différentes matières fondamentales liées à l'électronique (électronique analogique, électronique numérique, traitement du signal, automatique, ...). Ces notions abordées lors des enseignements théoriques sont souvent enseignées de manière cloisonnée. C'est pourquoi nous proposons aux étudiants des applications concrètes mettant en œuvre plusieurs matières au sein d'un même projet. Ainsi, pour compléter et illustrer les enseignements académiques, l'équipe d'enseignants en électronique et traitement du signal de l'ISAE a développé différents supports pédagogiques $[1,2,3,4,5$, 6] présentant des systèmes réels exploités dans le monde civil ou militaire (guidage radar, régulateur de vitesse adaptatif pour automobile, contrôle d'un véhicule électrique, ...).

Le nouveau projet décrit dans cet article est basé sur un système de géolocalisation en intérieur. Le but fixé aux étudiants est de calculer leur position à l'aide d'un récepteur situé dans une pièce équipée de plusieurs émetteurs de signaux à ultrason (satellites). Les étudiants disposent d'un récepteur à ultrason qui permet de recevoir simultanément les signaux émis par tous les satellites (cf. fig. 1). Le traitement des signaux reçus et le calcul de la position est réalisé sous Matlab $^{\circledR}$ par l'intermédiaire d'une carte d'acquisition.

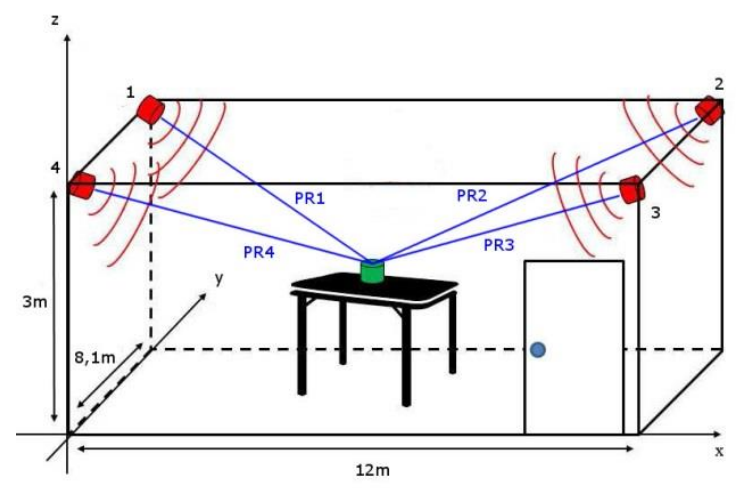

fig. 1 : Configuration minimale du système

\section{DESCRIPTION DU SUPPORT PEDAGOGIQUE}

La maquette de Géolocalisation en Intérieur par Ultrasons (GIU) est composée de 3 parties principales :

- La Constellation de Satellites : Celle-ci est composée de 8 émetteurs à ultrason large bande (Prowave 400 WB16). Les signaux sont générés à l'aide d'un Arduino $\mathrm{UNO}^{\circledR}$ à une fréquence porteuse de $40 \mathrm{KHz}$ modulée en phase à $4 \mathrm{KHz}$ (partie fournie aux étudiants).

- Le récepteur : Celui-ci est composé d'un récepteur à ultrason à large bande (SPM0204UD5). Le signal est filtré et amplifié de façon analogique. (partie fournie aux étudiants) 
- L'acquisition et le traitement : Celle-ci est composée d'une carte d'acquisition National Instruments ${ }^{\circledR}$ NiDaq $6064^{\mathrm{E}}$ et d'un PC équipé de Matlab ${ }^{\circledR}$ pour la partie traitement du signal et navigation (calcul de position).

\section{PRE REQUIS}

\subsection{Pré requis généraux}

$\mathrm{Ce}$ projet s'adresse aux élèves ingénieurs en fin de première année du cycle ingénieur. Ceux-ci possèdent les bases dans les domaines suivants :

- Electronique analogique (50 heures de formation: amplification, filtrage, oscillateurs)

- Systèmes/Traitement du signal $(50$ heures de formation: Laplace, Fourier, Bode, théorème de Shannon, transformée en Z, transformée de Fourier discrète, filtrage analogique et numérique)

- Automatique (30 heures de formation : modélisation et analyse des systèmes linéaires)

Pour réaliser ce projet, les étudiants doivent avoir quelques connaissances basiques du logiciel de calcul scientifique Matlab ${ }^{\circledR}$.

\subsection{Fonctionnement du système GIU}

\subsubsection{Principe de fonctionnement}

Les étudiants disposent d'une constellation de satellites émettant simultanément et de façon synchrone. Chaque satellite émet son propre code PRN (Pseudo Random Noise) ce qui permet de l'identifier. Dans le cas de cette maquette les satellites sont fixes et nous connaissons précisément leur position. Les distances à chaque satellite sont estimées par mesure du temps de vol de l'onde sonore dans l'air. On peut alors, par triangulation, calculer précisément l'endroit de réception du signal. La vitesse de propagation d'une onde acoustique étant connue ( $c \approx 340 \mathrm{~m} / \mathrm{s}$ dans l'air).

\subsubsection{Les signaux satellites}

Chaque satellite émet une porteuse (autour de $40 \mathrm{KHz}$ ) modulée en phase (modulation BPSK) par le code PRN comme illustré sur la figure 2. Les codes PRN que nous utilisons sont les vrais codes GOLD utilisés dans le système GPS [7, 8]. Il s'agit de séquences binaires périodiques (1023 bits ou chips). Ces séquences paraissent aléatoires mais sont parfaitement connues ; on dit qu'elles sont pseudo aléatoires.

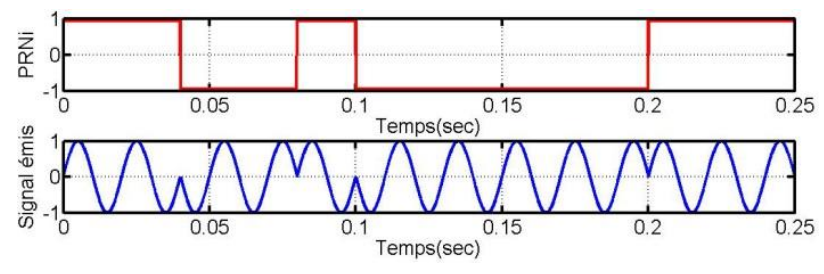

fig. 2 : Signal PRN(1), Signal modulé (2)

Cette modulation de phase associée à l'utilisation d'un PRN permet l'étalement du spectre du signal et ainsi d'augmenter (considérablement) le gain de traitement à la réception mais aussi et surtout de recevoir des données en Accès Multiple (plusieurs émetteurs utilisent la même fréquence porteuse, comme en télécommunications). Le gain à la réception est proportionnel à la largeur de la bande (Gain $=$ Bande $\times$ Temps d'intégration).

\subsubsection{Mesure du retard et calcul de pseudo-distance}

La mesure du temps de propagation de l'onde sonore dans l'air se fait par inter-corrélation entre le signal émis par le satellite et le même code généré au niveau du récepteur. Cependant, dû au fait que la base temps du récepteur n'est pas synchronisée avec celle des satellites, il est impossible de mesurer directement la distance qui sépare le récepteur d'un satellite. On mesure en réalité une pseudo-distance (ou Pseudo-Range, PR), biaisée par le décalage d'horloge $(\delta t)$ entre le récepteur et les satellites. Les pseudo-distances mesurées sont donc :

$$
P R_{i}=R_{i}+c \delta t
$$

Avec: $P R_{i} \quad$ Pseudo distance (mesurée) au satellite $i$

$R_{i} \quad$ Distance réelle au satellite $i$

$\delta t \quad$ décalage d'horloge entre le temps «satellites » et le temps « récepteur »

\subsubsection{Calcul du point (position en $X, Y, Z$ )}

Pour le calcul de la position du récepteur dans un espace en trois dimensions. Nous avons besoin de "voir" 4 satellites au minimum, car le décalage d'horloge ajoute une inconnue supplémentaire aux 3 inconnues spatiales. Nous obtenons un système d'au moins 4 équations à 4 inconnues :

$$
\begin{gathered}
\left(P R_{1}-c \delta t\right)=\sqrt{\left(X_{1}-X_{r}\right)^{2}+\left(Y_{1}-Y_{r}\right)^{2}+\left(Z_{1}-Z_{r}\right)^{2}} \\
\ldots \\
\left(P R_{i}-c \delta t\right)=\sqrt{\left(X_{i}-X_{r}\right)^{2}+\left(Y_{i}-Y_{r}\right)^{2}+\left(Z_{i}-Z_{r}\right)^{2}}
\end{gathered}
$$


Où

$\mathrm{Xr}, \mathrm{Yr}, \mathrm{Zr} \quad$ coordonnées du récepteur (3 inconnues)

$X_{i}, Y_{i}, Z_{i} \quad$ coordonnées du satellite $i$ (connues)

Ce système d'équations non-linéaires est alors résolu au sens des moindres carrés.

\section{TRAVAIL A REALISER}

Durant ce projet, les étudiants travaillent en binôme pendant 10 séances de 2 heures. La moitié, seulement, de ces séances sont encadrées par un enseignant. Ils doivent ainsi faire preuve d'autonomie dans la réalisation de leur projet. Suite à une première phase bibliographique durant laquelle les étudiants se familiarisent avec le fonctionnement $\mathrm{du}$ système GPS réel, ils doivent concevoir les programmes (sous Malab ${ }^{\circledR}$ ) permettant de calculer leur position à partir du signal recueilli par le récepteur qui leur est fourni. Pour cela, les étudiants doivent concevoir les fonctions principales suivantes, comme indiqué sur la figure 3. Comme dans le fonctionnement réel des récepteurs GPS, il existe 2 phases pour calculer sa position :

- La phase d'acquisition (appelée parfois « démarrage à froid ») qui consiste à identifier les satellites en visibilité et mesurer les retards de propagation et décalage doppler de leur signaux sans aucune information à priori.

- La phase de poursuite qui consiste simplement à estimer les nouveaux retards et décalage Doppler à chaque période de récurrence connaissant les anciens. Cette opération est réalisée à l'aide de boucle de contrôle.

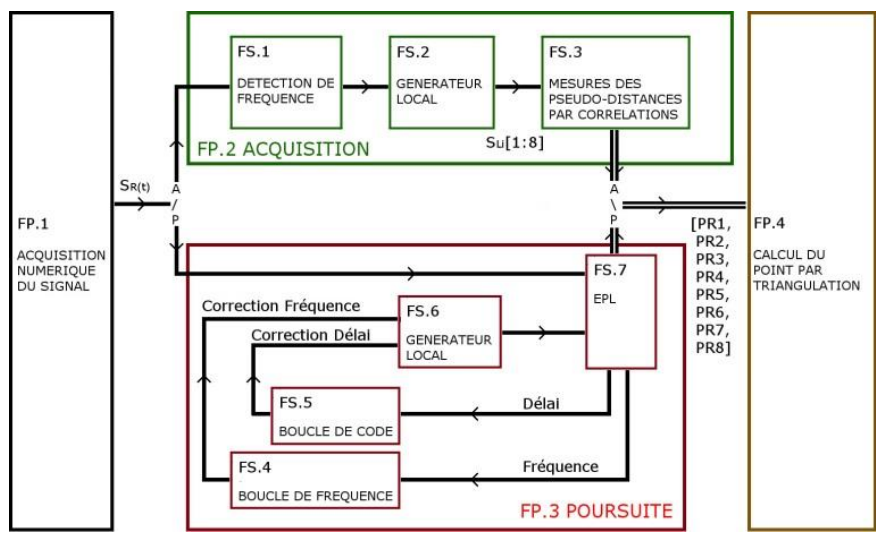

fig. 3 : Schéma Fonctionnel

\subsection{Numérisation du signal reçu}

Cette étape consiste simplement à acquérir le signal reçu avec la carte d'acquisition. La fréquence porteuse des émetteurs est de $40 \mathrm{KHz}$, avec une période de répétition de 0.25 secondes pour obtenir un code PRN complet (à $4 \mathrm{Kbits} / \mathrm{s})$. Nous proposons donc d'échantillonner et démoduler en même temps, par repliement du spectre, en utilisant une fréquence d'échantillonnage de $8 \mathrm{KHz}$. La fréquence porteuse reçue n'est cependant pas égale à $40 \mathrm{KHz}$ à cause des défauts électroniques à l'émission, d'un éventuel effet Doppler si le récepteur est mobile et $\mathrm{du}$ produit d'intermodulation entre le signal et la fréquence d'échantillonnage. On observe donc une modulation de fréquence qu'il faudra estimer et compenser lors de l'étape suivante (cf. fig. 4).

\subsection{Détection de fréquence}

Comme indiqué auparavant et comme cela est réalisé dans un récepteur GPS commercial, cette étape consiste à estimer la fréquence exacte du signal reçu (cf. fig. 4) de manière à générer, localement, un code identique et estimer le retard de propagation dans de bonnes conditions.

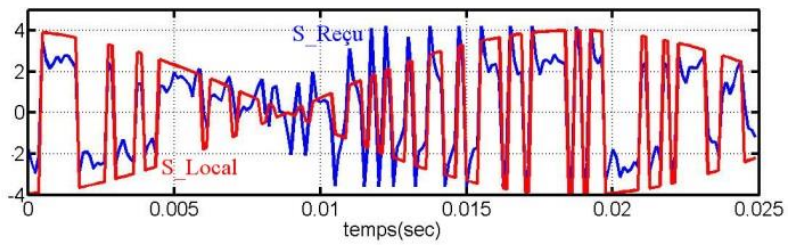

fig. 4 : Signal reçu $S_{R}(t)$ et Signal reconstitué $S_{L_{i}}(t)$

\subsection{Générateur local}

La réception simultanée des signaux émis par les satellites via un seul canal de réception, impose l'utilisation de la démodulation par corrélation. Pour réaliser cette opération il faut reproduire localement, au niveau du récepteur, les signaux émis par chacun des satellites, affectés des fréquences mesurées précédemment. Les étudiants doivent donc recréer sous Matlab $^{\circledR}$ les codes PR|N des satellites en visibilité, avant de procéder aux corrélations.

\subsection{Mesure des pseudo-distances}

Cette étape est le cœur du programme. L'inter-corrélation entre le signal reçu $S_{R}(t)$ et les signaux générés localement pour chaque satellite $S_{L_{i}}(t)$, permet d'estimer le décalage temporel entre le signal émis et le signal reçu.

$$
\operatorname{cor}_{S_{R}}^{S_{L i}}(k)=\sum_{n=0}^{1023} S_{L i}(n) * S_{R}(n-k)
$$


Comme nous l'avons vu précédemment, ce décalage est biaisé par le fait que les horloges d'émission et de réception ne peuvent pas être synchronisées. On obtient ainsi, non pas les distances réelles, mais des pseudodistances en multipliant ces retards de propagation par la célérité de l'onde.

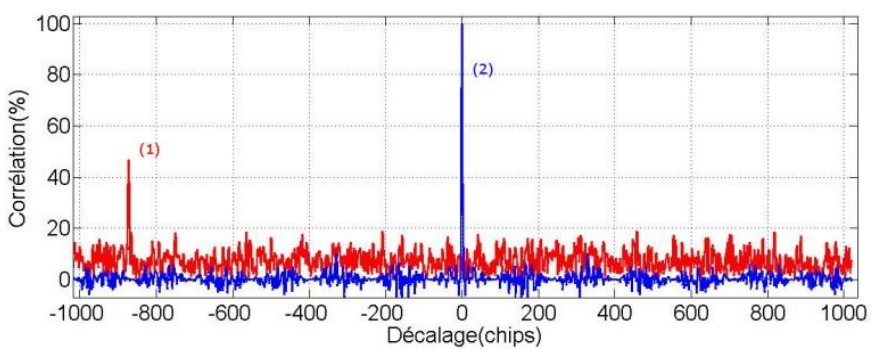

fig. 5 : Corrélation entre Signal local et Signal reçu (1), Autocorrélation du Signal local (2)

\subsection{Calcul du point}

Le calcul de point se fait en résolvant le système d'équation décrit à la partie 3.2.4. Dans notre cas, ce système d'équation ne peut pas être linéarisé, comme cela est fait pour le système GPS réel car les distances entre les satellites et les récepteurs sont beaucoup plus faibles. Les étudiants doivent donc résoudre ce système avec une méthode itérative de leur choix, vue en cours de math.

\subsection{Phase de poursuite}

Lorsqu'un satellite a été identifié et son retard et décalage Doppler mesuré, il n'est plus nécessaire de relancer une recherche globale de ces 2 paramètres à chaque étape. En effet, ceux-ci évoluent lentement et peuvent donc être poursuivis au cours du temps à l'aide de 2 boucles de contrôles imbriquées (cf. fig. 3), l'une pour la fréquence, l'autre pour le retard de propagation. C'est le rôle de cette phase de poursuite. Les étudiants doivent ici adapter les boucles de poursuite classiquement utilisées dans les systèmes GPS du commerce à leur fonctionnement propre. Ils doivent en particulier choisir l'ordre et la dynamique de ces boucles de manières à ce qu'elles soient le plus stable possible et ne décrochent pas.

\section{EXPLOITATION PEDAGOGIQUE}

La gestion du travail et le découpage des différentes taches du projet sont laissés au libre choix de chaque groupe. Une bonne conduite du projet est donc essentielle pour aboutir à une réalisation qui fonctionne. Les étudiants sont cependant guidés par la chronologie suivante :
1- Etude fonctionnelle (2h30). Cette première étape permet de définir précisément les différentes fonctions à réaliser en chiffrant les objectifs de chacune d'elles et les informations qu'elles doivent échanger.

2- Découpage du travail. Après avoir chiffré la charge de travail nécessaire à la réalisation de chaque fonction, cette étape permet au groupe en charge d'un projet de se répartir les tâches.

\section{3-Réalisation des différentes fonctions (10 h.).}

4-Association des sous programmes et test de l'ensemble (5h.).

5-Mesure des performances de leur système (2h30.).

6-Rédaction d'un rapport technique résumant le travail réalisé. Ce rapport est volontairement limité à 10 pages pour obliger les élèves à un travail de synthèse et de concision.

7-Présentation devant un jury du travail réalisé. Cette présentation de 15 minutes est suivie de 15 minutes de questions pour chaque groupe pendant lesquelles les étudiants doivent justifier leurs choix.

\section{RETOUR D'EXPERIENCE}

L'aspect ludique de ce type de projet et le fait qu'il soit très proche d'un système concret, connu de tous, amplifie la motivation des étudiants. On observe une certaine prise de conscience sur la réalité concrète des notions de base en automatique ou en traitement du signal. En particulier, les aspects temps réel, précision dans la résolution des systèmes d'équation, les notions de filtrage, de contrôle, de décrochage de boucle, de dynamique, de Doppler, de longueur d'onde sont perçus de manière plus naturelle, ce qui complète leurs connaissances parfois trop théoriques. De plus, ce type de projet permet aux étudiants de prendre un peu de recul sur les enseignements académiques présentés de façon disjointe et comprendre les analogies et interactions entre ces différentes matières.

Enfin la démarche proposée laisse une grande initiative dans le choix de la solution. Les étudiants doivent ainsi poser le problème avant de le résoudre, ce qui diffère grandement des exercices auxquels ils sont habitués lors des enseignements "classiques". Cette démarche de prise de décision est plus proche du travail qu'ils auront à accomplir dans leur futur métier d'ingénieur. 


\section{CONCLUSION}

De manière à illustrer la conception d'un système de traitement du signal complet (du capteur à l'information traitée), nous avons développé une maquette simplifiée d'un système de géolocalisation par satellite. Ce prototype est exploité au cours de projets de 20 heures par des étudiants travaillant en groupe dans le but d'estimer chacun sa position à l'aide de leur propre récepteur. Ce projet permet de décloisonner, de manière ludique, les différentes disciplines formant l'enseignement EEA et de mettre en relief les interactions entre ces différentes matières. Une part importante d'initiative est laissée aux étudiants dans le choix des solutions techniques à mettre en œuvre.

\section{Bibliographie}

[1] B. Mouton, F. Vincent, C. Nouals. "Réalisation d'un RADAR de poursuite", CETSIS 2003, Toulouse

[2] F. Vincent, B. Mouton, C. Nouals. " Outil pédagogique pour l'analyse des systèmes d'imagerie RADAR", CETSIS 2005, Nancy. Paru dans la revue J3eA - Vol. 4, Hors-série 2 - 32 (2005). EDP Sciences, 2005.

[3] F. Vincent, B. Mouton, C. Nouals. “Maquette pédagogique pour l'enseignement des systèmes radar", CETSIS 2007, Bordeaux. Paru dans la revue J3eA - EDP Sciences, 2008.

[4] F. Vincent, B. MOUTON, C. Nouals. "Contrôle d'un kart électrique à 2 moteurs indépendants”, CETSIS 2008, Bruxelles

[5] F. Vincent, M. Berranger, G. Beaugendre, R. Pascaud "Contrôle d'un système de tir balistique", CETSIS 2011, Trois Rivières, Canada, 23-26 Octobre 2011.

[6] V. Budinger, G. Beaugendre, M. Berranger, F. Vincent, R. Pascaud "Instrumentation d'une fusée à eau", CETSIS 2011, Trois Rivières, Canada, 23-26 Octobre 2011.

[7] E.D. Kaplan, C.J. Hegraty "Understanding GPS, principles and applications", ARTECH HOUSE INC, 2006.

[8] G. Bonin. "Système GPS de positionnement par satellite", "Technique de l'Ingénieur, traité Télécoms », Mai 2001. 